\title{
Bijections of dominant regions in the $m$-Shi arrangements of type $A, B$ and $C$
}

\author{
Myrto Kallipoliti $\llbracket$ and Eleni Tzanaki非 \\ ${ }^{1}$ Fak. für Mathematik, Universität Wien, Oskar-Morgenstern-Platz 1, 1090 Wien, Austria \\ ${ }^{2}$ Department of Applied Mathematics, University of Crete, 71409 Heraklion, Crete, Greece
}

\begin{abstract}
In the present paper, the relation between the dominant regions in the $m$-Shi arrangement of types $B_{n} / C_{n}$, and those of the $m$-Shi arrangement of type $A_{n-1}$ is investigated. More precisely, it is shown explicitly how the sets $\mathcal{R}^{m}\left(B_{n}\right)$ and $\mathcal{R}^{m}\left(C_{n}\right)$, of dominant regions of the $m$-Shi arrangement of types $B_{n}$ and $C_{n}$ respectively, can be projected to the set $\mathcal{R}^{m}\left(A_{n-1}\right)$ of dominant regions of the $m$-Shi arrangement of type $A_{n-1}$. This is done by using two different viewpoints for the representative alcoves of these regions: the Shi tableaux and the abacus diagrams. Moreover, bijections between the sets $\mathcal{R}^{m}\left(B_{n}\right), \mathcal{R}^{m}\left(C_{n}\right)$, and lattice paths inside a rectangle $n \times m n$ are provided.

Résumé. Dans cet article, nous étudions la relation entre les régions dominantes du $m$-arrangement de Shi de types $B_{n} / C_{n}$ et ceux du $m$-arrangement de Shi de type $A_{n-1}$. Plus précisément, nous montrons comment les ensembles $\mathcal{R}^{m}\left(B_{n}\right)$ et $\mathcal{R}^{m}\left(C_{n}\right)$, des régions dominantes du $m$-arrangement de Shi de types $B_{n}$ et $C_{n}$ respectivement, peuvent être projetés sur l'ensemble $\mathcal{R}^{m}\left(A_{n-1}\right)$ des régions dominantes du $m$-arrangement de Shi de types $A_{n-1}$. Pour cela nous utilisons deux points de vue différents sur les alcôves représentatives de ces régions: les tableaux de Shi et les diagrammes d'abaques. De plus, nous fournissons des bijections entre les ensembles $\mathcal{R}^{m}\left(B_{n}\right), \mathcal{R}^{m}\left(C_{n}\right)$, et les chemins à l'intérieur d'un rectangle $n \times m n$.
\end{abstract}

Keywords: Shi hyperplane arrengements, abacus diagram, affine permutations, lattice paths

\section{Introduction and Results}

The $m$-Shi arrangements associated to finite crystallographic root systems are well-studied hyperplane arrangements, with many connections to other combinatorial objects, such as parking functions and lattice paths, or even to other areas of mathematics, such as number theory, algebra and geometry. Before we proceed to the description of our results we provide the necessary definitions.

Let $V$ be a $d$-dimensional Euclidean space, with inner product $\langle\rangle,, \Phi$ a crystallographic root system spanning $V$, and let $m$ be a fixed nonnegative integer. The $m$-Shi arrangement associated to $\Phi$, denoted by $\operatorname{Shi}^{m}(\Phi)$, is the collection of hyperplanes in $V$ defined by the affine equations $\langle x, \alpha\rangle=k$, where $\alpha \in \Phi$ and $-m<k \leq m$. The connected components of the complement of $\operatorname{Shi}^{m}(\Phi)$ are called regions

\footnotetext{
$\dagger^{\dagger}$ mail: myrto.kallipoliti@univie.ac.at. Supported by the Special Research Programme "Algorithmic and Enumerative Combinatorics" (grant F50-N13).

‡Email: etzanaki@tem.uoc.gr.

1365-8050 @ 2015 Discrete Mathematics and Theoretical Computer Science (DMTCS), Nancy, France
} 
of the arrangement. The dominant chamber of $V$ is the intersection $\bigcap_{\alpha \in \Phi_{>0}}\{v \in V \mid\langle v, \alpha\rangle \geq 0\}$. Every region contained in the dominant chamber is called a dominant region. The set of all dominant regions in $\mathrm{Shi}^{m}(\Phi)$ is denoted by $\mathcal{R}^{m}(\Phi)$. Note that the dominant regions in the $m$-Shi arrangement $\mathrm{Shi}^{m}(\Phi)$ are the same as the dominant regions in the $m$-Catalan arrangement $\mathrm{Cat}^{(m)}(\Phi)$ [14, 15], and are counted by the generalized Catalan numbers

$$
N(\Phi, m)=\prod_{i=1}^{n} \frac{e_{i}+m h+1}{e_{i}+1},
$$

where where $n$ is the rank, $h$ is the Coxeter number and $e_{i}$ are the exponents of $\Phi$ [2]. In this work we are interested in the cases where $\Phi$ has type $A, B$ or $C$. In these cases, the corresponding generalized Catalan numbers reduce to the following well-known formulas:

$$
N\left(A_{n-1}, m\right)=\frac{1}{m n+1}\left(\begin{array}{c}
(m+1) n \\
n
\end{array}\right) \quad \text { and } \quad N\left(B_{n}, m\right)=N\left(C_{n}, m\right)=\left(\begin{array}{c}
(m+1) n \\
n
\end{array}\right) .
$$

The numbers $N\left(A_{n-1}, m\right)$, also known as Fuss-Catalan numbers, count an enormous number of combinatorial objects, such as $m$-Dyck paths of height $n$, while among the objects that the numbers $N\left(B_{n}, m\right)=N\left(C_{n}, m\right)$ count, are the lattice paths in a $n \times m n$ rectangle. The aim of the present paper is to explain the identity $N\left(B_{n}, m\right)=N\left(C_{n}, m\right)=(m n+1) N\left(A_{n-1}, m\right)$ by constructing "appropriate" bijections between the above mentioned objects. More precisely, our results are summarized as follows.

Theorem 1.1 There exist explicit bijections between the sets $\mathcal{R}^{m}\left(B_{n}\right), \mathcal{R}^{m}\left(C_{n}\right)$, and $\mathcal{R}^{m}\left(A_{n-1}\right) \times$ $\{1, \ldots, m n+1\}$.

Theorem 1.1 implies that there is a simple and explicit, yet not obvious, way to project the dominant regions of $\mathrm{Shi}^{m}\left(B_{n}\right)$ and $\mathrm{Shi}^{m}\left(C_{n}\right)$ to those of $\mathrm{Shi}^{m}\left(A_{n-1}\right)$. Another application of Theorem 1.1 is the following corollary.

Corollary 1.2 There exist explicit bijections between the sets $\mathcal{R}^{m}\left(B_{n}\right), \mathcal{R}^{m}\left(C_{n}\right)$, and lattice paths inside a rectangle $n \times m n$.

This paper is structured as follows. Section 2 includes background material on $m$-Shi arrangements. The notion of the dominant $m$-minimal alcoves is discussed and its connection to the dominant regions is explained. Moreover, two different viewpoints for the dominant $m$-minimal alcoves of type $A, B, C$ are described: The Shi tableau and the abacus bracket. Section 2.3 serves to clarify the relation between them. Theorem 1.1 and Corollary 1.2 are proven in Section 3 and Section 4 , respectively.

This work is an extended abstract of [9], and we have thus omitted most of the proofs.

\section{Preliminaries}

Let $\Phi$ be a crystallographic root system. The affine Shi arrangement $\operatorname{Shi}(\Phi)$ associated to $\Phi$ is the union of all hyperplanes of the form $H_{\alpha}^{r}=\{x \in V \mid\langle x, \alpha\rangle=r\}$, where $\alpha \in \Phi$ and $r \in \mathbb{Z}$. The connected components of the complement of this arrangement are called alcoves. Every alcove contained in the dominant chamber is called dominant. We denote by $\mathcal{A}(\Phi)$ the set of dominant alcoves of type $\Phi$. The fundamental alcove $A_{0}$ is the unique dominant alcove whose closure $\bar{A}_{0}$ contains the origin. For a fixed finite crystallographic root system $\Phi$, we denote by $S_{a}$ the set of reflections through all hyperplanes in 
Shi $(\Phi)$. The affine Weyl group $W_{a}$ of $\Phi$ is the infinite Coxeter group generated by the reflections in $S_{a}$. The group $W_{a}$ acts simply transitively on the set of alcoves in $\operatorname{Shi}(\Phi)$, thus one can identify each alcove $A$ with the unique $w \in W_{a}$ such that $A=w^{-1} A_{0}$. The above bijection restricts to a one between minimal length coset representatives in $W_{a} / W$ and dominant alcoves in Shi $(\Phi)$. In what follows we denote by $\mathcal{M}\left(W_{\alpha}\right)$ the set of minimal length coset representatives of $W_{\alpha} / W$. Thus we identify the sets $\mathcal{M}\left(W_{\alpha}\right)$ and $\mathcal{A}(\Phi)$.

Clearly, each dominant region $R \in \mathcal{R}^{m}(\Phi)$ consists of one or more alcoves. However, among all the alcoves contained in $R$, there exists a unique alcove $A_{R}$ which is closest to the origin. We call this the $m$-minimal alcove of $R$.

The main purpose of this section is to provide two different viewpoints of the dominant alcoves of the arrangements $\mathrm{Shi}^{m}\left(A_{n-1}\right), \mathrm{Shi}^{m}\left(B_{n}\right)$ and $\mathrm{Shi}^{m}\left(C_{n}\right)$ : (i) the Shi tableau and (ii) the abacus bracket. These combinatorial structures will be our tools for constructing our bijections.

\subsection{Combinatorial viewpoint I: The Shi tableau}

Based on an idea of Shi, who arranges the positive roots of $\Phi^{+}$in diagrams [12], we assign to each dominant alcove $A$ a set $\left\{k_{\alpha}, \alpha \in \Phi^{+}\right\} \subset \mathbb{N}$ of coordinates which describe the location of $A$ in the affine Shi arrangement Shi $\left(A_{n-1}\right)$. In particular, each $k_{\alpha}$ counts the number of integer translations of the hyperplane $H_{\alpha}$, which separate $A$ from the origin. The set of these coordinates, arranged according to the corresponding root diagram, is called the Shi tableau of the alcove.

We remark that the coordinates of a Shi tableau have to satisfy certain conditions. We present these conditions explicitly for the types $A, B$ and $C$ in the following paragraphs of this section, where $\varepsilon_{1}, \ldots, \varepsilon_{n+1}$ denotes the standard basis of $\mathbb{R}^{n+1}$. We refer to [2, 6, 11] for details.

\subsubsection{Type $A$}

In type $A_{n}$, a standard choice of positive and simple roots are the sets $\left\{\alpha_{i j}:=\varepsilon_{i}-\varepsilon_{j+1} \mid 1 \leq i \leq j \leq n\right\}$ and $\left\{\alpha_{i}:=\varepsilon_{i}-\varepsilon_{i+1} \mid 1 \leq i \leq n\right\}$, respectively. Each positive root can be written in terms of simple roots as:

$$
\alpha_{i j}=\alpha_{i}+\alpha_{i+1}+\cdots+\alpha_{j}, \text { for } 1 \leq i \leq j \leq n .
$$

Each Shi tableau in type $A_{n}$ can be seen as a staircase Young diagram of shape $(n, n-1, \ldots, 1)$ whose entries are non-negative integers $k_{i j}, 1 \leq i \leq j \leq n$ (see Figure 11. left). In particular, the coordinate $k_{i j}$ occupies the box $(i, n-j+1)$ (i) of the Shi tableau indicates the number of translations of the hyperplane $H_{\alpha_{i j}}$ that separate the alcove from the origin. The Shi conditions in this case are: for every $1 \leq i<j \leq n$ and all $i \leq \ell<j$

$$
k_{i j}=k_{i \ell}+k_{\ell+1 j}+\delta_{i \ell} \text { with } \delta_{i \ell} \in\{0,1\}
$$

\subsubsection{Type $B$ and $C$}

Traditionally, each Shi tableau of type $B$ or $C$ is represented in a shifted Young diagram. However, below we describe a different but equivalent way to write the diagram of coordinates. We do this to emphasize the relation of these types to the type $A$ case.

\footnotetext{
(i) The box $(i, j)$ lies in the $i$-th row and $j$-th column of the diagram, where rows are counted from top to bottom and columns from left to right.
} 


\begin{tabular}{|l|l|l|l|}
\hline$k_{14}$ & $k_{13}$ & $k_{12}$ & $k_{11}$ \\
\hline$k_{24}$ & $k_{23}$ & $k_{22}$ & \\
\hline$k_{34}$ & $k_{33}$ & \\
\cline { 1 - 2 }$k_{44}$ & \multicolumn{2}{|l}{} \\
\cline { 1 - 1 } &
\end{tabular}
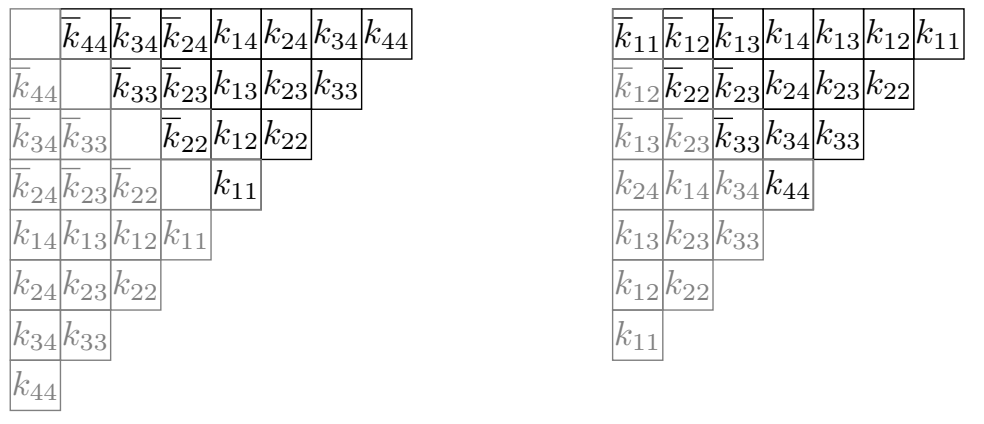

Fig. 1: The Shi tableau for type $A_{4}, B_{4}$ and $C_{4}$, respectively.

In type $B_{n}$, a standard choice of positive and simple roots are the sets $\left\{\varepsilon_{i}, \varepsilon_{n}, \varepsilon_{i} \pm \varepsilon_{j+1} \mid 1 \leq i \leq j \leq\right.$ $n-1\}$ and $\left\{\alpha_{i}:=\varepsilon_{i}-\varepsilon_{i+1}, \alpha_{n}:=\varepsilon_{n} \mid 1 \leq i \leq n-1\right\}$ respectively. If, for $1 \leq i \leq j \leq n-1$, we set $\alpha_{i j}:=\varepsilon_{i}-\varepsilon_{j+1}, \alpha_{i n}:=\varepsilon_{i}$ and $\bar{\alpha}_{i j}=\varepsilon_{i}+\varepsilon_{j+1}$, we can then write:

$$
\begin{aligned}
& \alpha_{i j}=\alpha_{i}+\cdots+\alpha_{j}, \text { for } 1 \leq i \leq j \leq n, \text { and } \\
& \bar{\alpha}_{i j}=\alpha_{i}+\cdots+\alpha_{j}+2\left(\alpha_{j+1}+\cdots+\alpha_{n-1}+\alpha_{n}\right) .
\end{aligned}
$$

We arrange the coordinates in a staircase diagram of shape $(2 n, 2 n-1, \ldots, 1)$ as follows: for each $1<i \leq j \leq n$ the coordinate $k_{i j}$ occupies the box $(n-j+1, n-i+2)$, whereas for each $1 \leq i \leq j \leq n$ the coordinate $k_{i j}$ occupies the box $(n-j+1, n+i)$. We finally fill the remaining boxes so that the diagram is self-conjugate. The boxes $(i, i)$ are left empty. Notice that we can fill appropriately the empty boxes so that the tableau obtained is in fact a (self-conjugate) Shi tableaux of type $A_{2 n}$.

In type $C_{n}$, a standard choice of positive and simple roots are the sets: $\left\{2 \varepsilon_{i}, 2 \varepsilon_{n}, \varepsilon_{i} \pm \varepsilon_{j+1} \mid 1 \leq i \leq\right.$ $j \leq n-1\}$ and $\left\{\alpha_{i}:=\varepsilon_{i}-\varepsilon_{i+1}, \alpha_{n}:=2 \varepsilon_{n} \mid 1 \leq i \leq n-1\right\}$ respectively. If, for $1 \leq i \leq j \leq n-1$, we set $\alpha_{i j}:=\varepsilon_{i}-\varepsilon_{j+1}, \alpha_{i n}:=\varepsilon_{i}+\epsilon_{n}$ and $\bar{\alpha}_{i j}:=\varepsilon_{i}+\varepsilon_{j+1}$, we can then write:

$$
\begin{aligned}
& \alpha_{i j}=\alpha_{i}+\cdots+\alpha_{j}, \text { for } 1 \leq i \leq j \leq n, \text { and } \\
& \bar{\alpha}_{i j}=\alpha_{i}+\cdots+\alpha_{j}+2\left(\alpha_{j+1}+\cdots+\alpha_{n-1}\right)+\alpha_{n} .
\end{aligned}
$$

We arrange the coordinates in a staircase diagram of shape $(2 n-1,2 n-2, \ldots, 1)$ as follows: for $1 \leq$ $i \leq j \leq n-1$ the coordinate $\bar{k}_{i j}$ occupies the box $(i, j)$, whereas for $1 \leq i \leq j \leq n$ the coordinate $k_{i j}$ occupies the box $(i, 2 n-j)$. We finally fill the remaining boxes so that the diagram is self-conjugate. Notice that the tableau obtained this way is in fact a (self-conjugate) Shi tableaux of type $A_{2 n-1}$.

Tableaux can also be used to encode dominant regions in the Shi arrangement $\operatorname{Shi}^{m}(\Phi)$. The Shi tableau for a dominant region $R$ in $\operatorname{Shi}^{m}(\Phi)$ is the set $\left\{r_{\alpha}, \alpha \in \Phi^{+}\right\} \subset \mathbb{N}$ of coordinates, where $r_{\alpha}$ counts the number of integer translates $H_{\alpha, k}$ of $H_{\alpha}$, that separate $R$ from the origin. By definition of $\operatorname{Shi}^{m}(\Phi)$, it is immediate that $0 \leq r_{\alpha} \leq m$.

As we mentioned in the first paragraph of Section 2, each dominant region in $\operatorname{Shi}^{m}(\Phi)$ is uniquely represented by its $m$-minimal alcove. One can switch from the tableau of the region $R$ to that of its $m$-minimal alcove $A_{R}$ and vice versa, as indicated by the following lemma (see also Figure 2 ). 
Lemma 2.1 Let $R$ be a dominant region in $\mathrm{Shi}^{m}(\Phi)$ with m-minimal alcove $A_{R}$ Let also $T_{R}=\left\{r_{\alpha}\right.$ : $\left.\alpha \in \Phi^{+}\right\}$and $T=\left\{k_{\alpha}: \alpha \in \Phi^{+}\right\}$be the Shi tableau of $R$ and $A_{R}$ respectively. Then, for each $\alpha \in \Phi^{+}$ the following relations hold:

(i) $r_{\alpha}=\min \left\{m, k_{\alpha}\right\}$, and

(ii) $k_{\alpha}=\sum_{i=1}^{n} c_{i} r_{\alpha_{i}}$, where $\sum_{i=1}^{n} c_{i} \alpha_{i}$ is the expression of $\alpha$ as a (nonnegative) linear combination of simple roots.

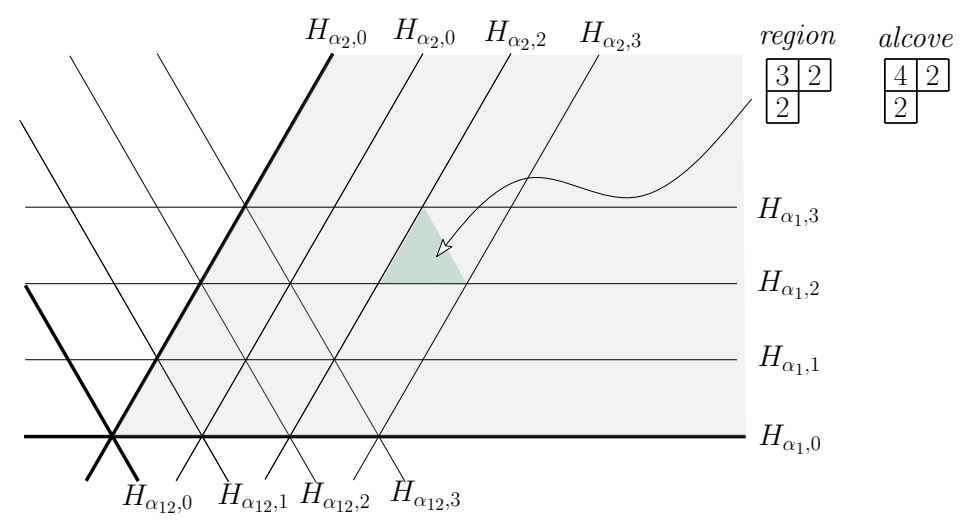

Fig. 2: The arrangement $\operatorname{Shi}^{3}\left(A_{2}\right)$. The green alcove is a 3 -minimal alcove. Its Shi tableau together with the Shi tableau of the region where it belongs, are shown on the right.

\subsection{Combinatorial viewpoint II: The abacus bracket}

\subsubsection{Type $A$}

A standard way to realize the affine group $\widetilde{A}_{n-1}$ is by identifying it with the set of all $\mathbb{Z}$-permutations, which are bijections $w$ of $\mathbb{Z}$ in itself such that: $w(x+n)=w(x)+n$ for all $x \in \mathbb{Z}$, and $\sum_{i=1}^{n} w(i)=$ $\left(\begin{array}{c}n+1 \\ 2\end{array}\right)$, with composition as group operation. Since such an element $w$ is uniquely determined by its values on the set $\{1, \ldots, n\}$, we use the base window notation of $w$ i.e., we write $w=[w(1), \ldots, w(n)]$. In this paper we will often write the base window of a permutation $w \in \widetilde{A}_{n-1}$ in a more concise form, which is actually equivalent to its abacus representation [3, 5]. To do this, for each $a, \ell \in \mathbb{Z}$ we denote by $a^{\ell}$ the sum $a+n \ell$ and we say that $\ell$ is the level of $a$. Then, if $w(i)=r_{i}^{\ell_{i}}$, conditions (i) and (ii) imply that we can write each $w \in \widetilde{A}_{n-1}$ as $w=\left[r_{1}^{\ell_{1}}, \ldots, r_{n}^{\ell_{n}}\right]$ where $\left\{r_{1}, \ldots, r_{n}\right\}=\{1, \ldots, n\}$ and $\ell_{1}+\cdots+\ell_{n}=0$. We say that $\left[r_{1}^{\ell_{1}}, \ldots, r_{n}^{\ell_{n}}\right]$ is the abacus bracket or simply bracket of $w$.

If, in addition, the entries of the base window are sorted i.e., $w(1)<\cdots<w(n)$, then $w \in \mathcal{M}\left(\widetilde{A}_{n-1}\right)$. In fact, it turns out that all such $w \in \widetilde{A}_{n-1}$ biject to the set of minimal length coset representatives of $\widetilde{A}_{n-1} / A_{n-1}$ (see [4, Chapter 8.3]). Since the sets $\mathcal{A}\left(A_{n-1}\right)$ and $\mathcal{M}\left(\widetilde{A}_{n-1}\right)$ are in bijection, when we write $w=[w(1), \ldots, w(n)] \in \mathcal{A}\left(A_{n-1}\right)$ we mean that $w$ is a dominant alcove that corresponds to the minimal length coset representative $[w(1), \ldots, w(n)]$ with $w(1)<\cdots<w(n)$. In this special case we can alternatively encode $w$ by its vector of levels $\vec{n}(w)=\left(\beta_{1}, \ldots, \beta_{n}\right)$ i.e., the vector whose $i$-th 
component $\beta_{i}$ is equal to the level of the unique $w(j)$ of the base window with $w(j)=i \bmod n$. For instance, if $w$ has base window $[-6,-4,1,19]$, then its abacus bracket is $\left[2^{-2}, 4^{-2}, 1^{0}, 3^{4}\right]$, and its vector of levels is $\vec{n}(w)=(0,-2,4,-2)$.

\subsubsection{Type $B$ and $C$}

We recall that the Coxeter group $\widetilde{B}_{n}$ is a subgroup of $\widetilde{C}_{n}$, and thus every element of $\widetilde{B}_{n}$ lies also in $\widetilde{C}_{n}$. Now, a standard way to describe the affine group $\widetilde{C}_{n}$ is by identifying it with the set of all mirrored $\mathbb{Z}$ permutations, which are bijections $w$ of $\mathbb{Z}$ in itself such that: $w(x+N)=w(x)+N$ and $w(-x)=$ $-w(x)$ for all $x \in \mathbb{Z}$, where $N=2 n+1$ and with composition as group operation. Combining the above conditions, one can easily see that $\sum_{i=1}^{2 n+1} w(i)=\left(\begin{array}{c}2 n+2 \\ 2\end{array}\right)$ and thus $\widetilde{C}_{n}$ is a subgroup of $\widetilde{A}_{2 n+1}$. A permutation $w \in \widetilde{C}_{n}$ is completely determined by its values on $\{1, \ldots, n\}$. However, for reasons that will become apparent later, we will identify each permutation $w \in \widetilde{C}_{n}$ with the ordered sequence $w=[w(1), \ldots, w(2 n)]$, which we will also call the base windon [ii) of $w$. Similar to type $A$, the bracket of $w \in \widetilde{C}_{n}$ can be written in the concise form $\left[r_{1}^{\ell_{1}}, \ldots, r_{2 n}^{\ell_{2 n}}\right]$, where now $r^{\ell}:=r+\ell N$. Again, one can easily see that $\left\{r_{1}, \ldots, r_{2 n}\right\}=\{1, \ldots, 2 n\}, \ell_{1}+\cdots+\ell_{2 n}=0$ and $\ell_{i}=-\ell_{j}$ if $r_{i}+r_{j}=N$. Finally, $w \in \mathcal{M}\left(\widetilde{C}_{n}\right)$ if and only if $w(1)<\cdots<w(n+1)$. See also [4, Chapter 8.4,8.5][8].

\subsection{From the Shi tableau to the abacus bracket and vice versa}

In [6, Section 2.7] Fishel et al., by rephrasing results of Shi [13], related the two different viewpoints of Sections 2.1 (Shi tableau and abacus bracket). In particular, they proved the following two propositions.

Proposition 2.2 Let $w=\left[r_{1}^{\ell_{1}}, \ldots, r_{n}^{\ell_{n}}\right] \in \mathcal{A}\left(A_{n-1}\right)$. We define $f(w)$ to be the Young tableau of shape $(n-1, n-2, \ldots, 1)$ with entries $k_{i j}, 1 \leq i \leq j \leq n-1$, where:

$$
k_{i j}=\left\{\begin{array}{lll}
\ell_{j+1}-\ell_{i}, & \text { if } & r_{i}<r_{j+1} \\
\ell_{j+1}-\ell_{i}-1, & \text { if } & r_{i}>r_{j+1} .
\end{array}\right.
$$

The map $f$ is a bijection between the set $\mathcal{A}\left(A_{n-1}\right)$ and the set of Shi tableaux of type $A_{n-1}$. (iii)

Proposition 2.3 Let $T=\left\{k_{i j}: 1 \leq i \leq j \leq n\right\}$ be the Shi tableau of a dominant alcove. For each $1 \leq j \leq n$ let $b_{j}:=\left|\left\{(1, \ell, j), 1 \leq \ell<j: k_{1, j}=k_{1, \ell}+k_{\ell+1, j}+1\right\}\right|$. Let also $\sigma=[\sigma(1), \ldots, \sigma(n)]$ be the permutation of $\{1, \ldots, n\}$ with inversion table $\left(b_{1}, \ldots, b_{n}\right)$ (iv) We define $g(T)$ to be the balanced bracket corresponding to the one that has vector of levels

$$
\left(0, k_{1, \sigma(1)}, k_{1, \sigma(2)}, \ldots, k_{1, \sigma(n)}\right) .
$$

The map $g$ is a bijection between the set of Shi tableaux of type $A_{n-1}$ and the set $\mathcal{A}\left(A_{n-1}\right)$.

Example 2.4 The dominant alcove $w=\left[4^{-3}, 1^{-1}, 2^{2}, 3^{2}\right]$ has vector of levels $(-1,2,2,-3)$. Below are the Shi tableau of $w$ and of the dominant region in $\mathcal{R}^{3}\left(A_{3}\right)$ that it represents.

(ii) Notice that if we wanted to be consistent with the definition of the base window for $\widetilde{A}_{2 n+2}$, we would also have to add the value $w(0)$ in the bracket. Since however condition (ii) implies that $w(0)=0$, we miss no information by ignoring this value.

(iii) We also use the formula of Equation 2 to map elements of $\mathcal{A}\left(C_{n}\right)$ to Shi tableaux of type $C_{n}$.

(iv) Given a permutation $\sigma$ of $\{1, \ldots, n\}$, its inversion table is a length $n$ sequence $b_{1}, \ldots, b_{n}$, where $b_{i}$ is the number of elements that are smaller than $i$ and appear to the right of $i$ in $\sigma$. 


\begin{tabular}{|l|l|l|}
\hline 4 & 4 & 1 \\
\hline 3 & 3 & \\
\cline { 1 - 1 } 0 & \multicolumn{2}{|l}{} \\
\hline
\end{tabular}

The following theorem is a rephrasing of [1, Theorem 4.5], and therefore the proof is omitted.

Theorem 2.5 Let $w$ be a dominant alcove in $\operatorname{Shi}\left(A_{n-1}\right)$ with vector of levels $\vec{n}(w)=\left(\beta_{1}, \ldots, \beta_{n}\right)$. Then, $w$ is the m-minimal alcove of a dominant region $\mathcal{R} \in \mathrm{Shi}^{m}\left(A_{n-1}\right)$ if and only if

(i) $\beta_{i+1}-\beta_{i} \leq m$ for all $1 \leq i \leq n-1$, and

(ii) $\beta_{1}-\beta_{n}-1 \leq m$.

\section{The projection}

In this section we prove Theorem 1.1 Let $w=[w(1), \ldots, w(n)] \in \mathcal{M}\left(\widetilde{A}_{n-1}\right)$ and $k \in \mathbb{Z}$. In what follows, we denote by $w_{k}$ the sequence $(w(1)+k, \ldots, w(n)+k)$. We remark that $w_{k}$ does not necessarily define a minimal coset representative in $\tilde{A}_{n-1} / A_{n-1}$, however, if we expand it appropriately, we can obtain minimal coset representatives in $\tilde{A}_{2 n-1} / A_{2 n-1}$ and $\tilde{A}_{2 n} / A_{2 n}$. More precisely, we have the following definitions which will be crucial for constructing the bijections of Theorem 1.1

Definition 3.1 Let $w \in \mathcal{M}\left(\widetilde{A}_{n-1}\right)$ with vector of levels $\vec{n}(w)=\left(\beta_{1}, \ldots, \beta_{n}\right)$. The type $C$ antisymmetric expansion of $w$, denoted by $\alpha^{C}(w)$, is the minimal coset representative in $\widetilde{A}_{2 n-1} / A_{2 n-1}$ whose vector of levels is $\left(\beta_{1}, \ldots, \beta_{n},-\beta_{n}, \ldots,-\beta_{1}\right)$.

Definition 3.2 Let $w \in \mathcal{M}\left(\widetilde{A}_{n-1}\right)$ with vector of levels $\vec{n}(w)=\left(\beta_{1}, \ldots, \beta_{n}\right)$. The type $B$ antisymmetric expansion of $w$, denoted by $\alpha^{B}(w)$, is the minimal coset representative in $\widetilde{A}_{2 n} / A_{2 n}$ whose vector of levels is $\left(\beta_{1}, \ldots, \beta_{n}, 0,-\beta_{n}, \ldots,-\beta_{1}\right)$.

\subsection{Proof of Theorem 1.1}

We first focus on type $C$.

Proposition 3.3 If $w \in \mathcal{A}\left(A_{n-1}\right)$ and $k \in \mathbb{Z}$, then $\alpha^{C}\left(w_{k}\right) \in \mathcal{A}\left(C_{n}\right)$. Conversely, every alcove in $\mathcal{A}\left(C_{n}\right)$ can be written in the form $\alpha^{C}\left(w_{k}\right)$ for some $w \in \mathcal{A}\left(A_{n-1}\right)$ and $k \in \mathbb{Z}$.

Proof: In view of [8, Lemma 2.5] the first part of the statement is a direct consequence of Definition 3.1 Let now $w \in \mathcal{A}\left(C_{n}\right)$ with abacus bracket $\left[r_{1}^{\ell_{1}}, \ldots, r_{2 n}^{\ell_{2 n}}\right]$, where $r_{i}^{\ell_{i}}=r_{i}+N \ell_{i}$. We consider the sequence $\left(r_{i_{1}}^{\ell_{i_{1}}}, \ldots, r_{i_{n}}^{\ell_{i_{n}}}\right)$, where $r_{i_{j}} \in\{1, \ldots, n\}$. Let $k=\sum_{j=0}^{n} \ell_{i_{j}}$. We write $-k=r+n \ell$, for some $r \in\{1, \ldots, n\}$ and $\ell \in \mathbb{Z}$. For $j \in\{1, \ldots, n\}$ we set

$$
\bar{r}_{j}^{\bar{\ell}_{j}}= \begin{cases}\left(r_{i_{j}}+r\right)^{\left(\ell_{i_{j}}+\ell\right)}, & \text { if } r_{i_{j}}+r \leq n \\ \left(\left(r_{i_{j}}+r\right) \bmod n\right)^{\left(\ell_{i_{j}}+\ell\right)+1}, & \text { otherwise. }\end{cases}
$$

Then, the ordered sequence of $\bar{r}_{j}^{\bar{\ell}_{j}}$ defines an abacus bracket, and let $\bar{w} \in \mathcal{A}\left(A_{n-1}\right)$ denote its corresponding alcove. It is immediate then the fact that $w=\alpha^{C}\left(\bar{w}_{k}\right)$. 
Corollary 3.4 The map $\alpha^{C}: \mathcal{A}\left(A_{n-1}\right) \times \mathbb{Z} \rightarrow \mathcal{A}\left(C_{n}\right)$, defined by $\alpha^{C}(w, k)=\alpha^{C}\left(w_{k}\right)$, is a bijection.

Let $w$ be an $m$-minimal dominant alcove in $\operatorname{Shi}^{m}\left(A_{n-1}\right), k \in \mathbb{Z}$ and consider the antisymmetric expansion $\alpha^{C}\left(w_{k}\right)$. The following question arises naturaly:

Question 1 For which choices of $k \in \mathbb{Z}$ is the dominant alcove $\alpha^{C}\left(w_{k}\right)$ of $\operatorname{Shi}^{m}\left(C_{n}\right)$ m-minimal?

The next proposition provides the necessary conditions for the number $k$ in order $\alpha^{C}\left(w_{k}\right)$ to be an $m$-minimal alcove in $\operatorname{Shi}^{m}\left(C_{n}\right)$.

Proposition 3.5 Let $w$ be an m-minimal alcove with $\vec{n}(w)=\left(\beta_{1}, \ldots, \beta_{n}\right)$, and let $k=r+n \ell$, where $r \in\{1,2, \ldots, n\}$ and $\ell \in \mathbb{Z}$. Then, $\alpha^{C}\left(w_{k}\right)$ is m-minimal if the following conditions hold.

- $-\left\lfloor\frac{m}{2}\right\rfloor-1-\beta_{n} \leq \ell \leq\left\lfloor\frac{m-1}{2}\right\rfloor-\beta_{1}$ and $r=n$, or

- $-\beta_{n-r}-\left\lfloor\frac{m}{2}\right\rfloor \leq \ell \leq-\beta_{n-r+1}+\left\lfloor\frac{m-1}{2}\right\rfloor$, if $r<n$.

Proof: Suppose first that $r=n$. Then $\vec{n}\left(w_{k}\right)=\left(\beta_{1}+\ell+1, \ldots, \beta_{n}+\ell+1\right)$. It suffices to check the criterion of Theorem 2.5 for the following two pairs of consecutive $\bmod 2 n$ integers: $\left((2 n)^{-\beta_{1}-\ell-1}, 1^{\beta_{1}+\ell+1}\right)$, and $\left(n^{\beta_{n}+\ell+1},(n+1)^{-\beta_{n}-\ell-1}\right)$. For the first pair we must have $\beta_{1}+\ell+1-\left(-\beta_{1}-\ell-1\right)-1 \leq m \Rightarrow$ $\ell \leq \frac{m-1}{2}-\beta_{1}$, while for the second pair we must have $-\beta_{n}-\ell-1-\left(\beta_{n}+\ell+1\right) \leq m \Rightarrow \ell \geq-\frac{m}{2}-1-\beta_{n}$. Combining the two inequalities, and since $\ell$ is an integer, we deduce that

$$
-\left\lfloor\frac{m}{2}\right\rfloor-1-\beta_{n} \leq \ell \leq\left\lfloor\frac{m-1}{2}\right\rfloor-\beta_{1} .
$$

Suppose now that $r<n$. Then $\vec{n}\left(w_{k}\right)=\left(\beta_{n-r+1}+\ell+1, \ldots, \beta_{n}+\ell+1, \beta_{1}+\ell, \beta_{2}+\ell, \ldots, \beta_{n-r}+\ell\right)$. It suffices to check the criterion of Theorem 2.5 for the following two pairs of consecutive $\bmod 2 n$ integers: $\left((2 n)^{-\beta_{n-r+1}-\ell-1}, 1^{\beta_{n-r+1}+\ell+1}\right)$, and $\left(n^{\beta_{n-r}+\ell},(n+1)^{-\beta_{n-r}-\ell}\right)$. For the first pair we must have $\beta_{n-r+1}+\ell+1-\left(-\beta_{n-r+1}-\ell-1\right)-1 \leq m \Rightarrow \ell \leq-\beta_{n-r+1}+\frac{m-1}{2}$, while for the second pair we must have $-\beta_{n-r}-\ell-\left(\beta_{n-r}+\ell\right) \leq m \Rightarrow \ell \geq-\beta_{n-r}-\frac{m}{2}$. Combining the two inequalities, and since $\ell$ is an integer, we deduce that

$$
-\beta_{n-r}-\left\lfloor\frac{m}{2}\right\rfloor \leq \ell \leq-\beta_{n-r+1}+\left\lfloor\frac{m-1}{2}\right\rfloor .
$$

Let $w$ be an $m$-minimal alcove. By counting the possible values of $k$ that satisfy the conditions of Lemma 3.5. we obtain the following corollary.

Corollary 3.6 For each m-minimal alcove $w$ in $\mathrm{Shi}^{m}\left(A_{n-1}\right)$ there exist exactly $m n+1$ distinct integer values for $k \in \mathbb{Z}$ such that $\alpha^{C}\left(w_{k}\right)$ is an m-minimal alcove in $\operatorname{Shi}^{m}\left(C_{n}\right)$.

Notation. Let $w$ be an $m$-minimal alcove. We denote by $K_{m}(w)$ the set of integers $k$ for which the alcove $\alpha^{C}\left(w_{k}\right)$ is $m$-minimal as well.

We are now in position to describe the bijection $\phi: \mathcal{R}^{m}\left(C_{n}\right) \rightarrow \mathcal{R}^{m}\left(A_{n-1}\right) \times\{1, \ldots, m n+1\}$. Let $R \in \mathcal{R}^{m}\left(C_{n}\right)$, and let $w_{R}$ be its representative ( $m$-minimal) alcove. Proposition 3.3 implies that there is an alcove $w \in \mathcal{A}\left(A_{n-1}\right)$ such that $\alpha^{C}\left(w_{k^{*}}\right)=w_{R}$ for some $k^{*} \in \mathbb{Z}$. Let $k_{1}<\cdots<k_{m n+1}$ be the elements of $K_{m}(w)$. Since $k^{*} \in K_{m}(w)$, there is an index $i \in\{1, \ldots, m n+1\}$ for which $k_{i}=k^{*}$. We set $\phi(R)=(w, i)$. We leave it to the reader to check that $\phi$ defines a bijection between the sets $\mathcal{R}^{m}\left(C_{n}\right)$ and $\mathcal{R}^{m}\left(A_{n-1}\right) \times\{1, \ldots, m n+1\}$. 
Corollary 3.7 The map $\bar{\phi}: \mathcal{R}^{m}\left(C_{n}\right) \rightarrow \mathcal{R}^{m}\left(A_{n-1}\right)$, defined by $\bar{\phi}(R)=\operatorname{pr}_{1}(\phi(R))$ is a surjection.

We now focus on type $B$ and follow the same steps. The main difference is that the antisymmetric expansion of type $B$ defines a surjection and not a bijection (see Proposition 3.9 below). This is however enough for the construction of a bijection between the sets $\mathcal{R}^{m}\left(B_{n}\right)$ and $\mathcal{R}^{m}\left(A_{n-1}\right) \times\{1, \ldots, m n+1\}$. A crucial remark constitutes the following proposition.

Proposition 3.8 Let $w$ be an m-minimal dominant alcove in $\operatorname{Shi}^{m}\left(A_{n-1}\right)$. Then for every $k \in K_{m}(w)$, the alcove $\alpha^{B}\left(w_{k}\right)$ is m-minimal in $\operatorname{Shi}^{m}\left(A_{2 n}\right)$.

Proof (sketch): We consider first the type- $C$ antisymmetric expansion $\alpha^{C}\left(w_{k}\right)$. Its vector of levels can be written as $\vec{n}\left(\alpha^{C}\left(w_{k}\right)\right)=\left(\beta_{1}, \ldots, \beta_{n},-\beta_{n}, \ldots,-\beta_{1}\right)$. Remark that $\beta_{n} \geq 0$. Then it follows from the definitions of type $B$ and $C$ antisymmetric expansions that $\vec{n}\left(\alpha^{B}\left(w_{k}\right)\right)=\left(\beta_{1}, \ldots, \beta_{n}, 0,-\beta_{n}, \ldots,-\beta_{1}\right)$. Since $\alpha^{C}\left(w_{k}\right)$ is already an $m$-minimal alcove, it suffices to check the conditions of Theorem 2.5 for the following two pairs of consecutive $\bmod N$ integers: $\left(n^{\beta_{n}},(n+1)^{0}\right)$, and $\left((n+1)^{0},(n+2)^{-\beta_{n}}\right)$. Clearly both pairs reduce to the inequality $-\beta_{n} \leq m$, which holds since $\beta_{n} \geq 0$ and $m \geq 1$.

For a $2 n$-staircase Young tableau $T=\left\{k_{i j}\right\}$ we denote by $\bar{T}=\left\{\bar{k}_{i j}\right\}$ the Young tableau obtained by deleting for every $i=1, \ldots, 2 n$ the entry of the box $(i, i)$. The following is a weak type- $B$ analogue of Proposition 3.3 and Corollary 3.4

Proposition 3.9 The map $\alpha^{B}: \mathcal{A}\left(A_{n-1}\right) \times \mathbb{Z} \rightarrow \mathcal{A}\left(B_{n}\right)$, defined by $\alpha^{B}(w, k)=\bar{f}\left(\alpha^{B}\left(w_{k}\right)\right)$, where $f$ is the bijection of Proposition 2.2 is a surjection.

Proof (sketch): Let $(w, k) \in \mathcal{A}\left(A_{n-1}\right) \times \mathbb{Z}$. First we note that it is an immediate consequence of Definition 3.2 that the tableau $f\left(\alpha^{B}\left(w_{k}\right)\right)$ is self-conjugate Shi tableau of type $A_{2 n}$, thus the map $\bar{f}\left(\alpha^{B}\left(w_{k}\right)\right)$ is well-defined. We will show now that $\alpha^{B}$ is surjective. Let $T$ be the Shi tableau of some dominant alcove in $\mathcal{A}\left(B_{n}\right)$. Then there is a tableau $T^{\prime}$ of type $A_{2 n}$ that is obtained from $T$ by filling the diagonal boxes. We recall Proposition 2.3 and consider the corresponding dominant alcove, $g\left(T^{\prime}\right)=\left[r_{1}^{\ell_{1}}, \ldots, r_{2 n+1}^{\ell_{2 n+1}}\right]$. Note that $r_{n+1}^{\ell_{n+1}}=(n+1)^{0}$. Following the steps of Proposition 3.3. consider the sequence $\left(r_{i_{1}}^{\ell_{i_{1}}}, \ldots, r_{i_{n}}^{\ell_{i_{n}}}\right)$, where $r_{i_{j}} \in\{1, \ldots, n\}$. Let $k=\sum_{j=0}^{n} \ell_{i_{j}}$. We write $-k=r+n \ell$, for some $r \in\{1, \ldots, n\}$ and $\ell \in \mathbb{Z}$. For $j \in\{1, \ldots, n\}$ we set

$$
\bar{r}_{j}^{\bar{\ell}_{j}}= \begin{cases}\left(r_{i_{j}}+r\right)^{\left(\ell_{i_{j}}+\ell\right)}, & \text { if } r_{i_{j}}+r \leq n, \\ \left(\left(r_{i_{j}}+r\right) \bmod n\right)^{\left(\ell_{i_{j}}+\ell\right)+1}, & \text { otherwise. }\end{cases}
$$

Then, the ordered sequence of $\bar{r}_{j}^{\bar{\ell}_{j}}$ defines an abacus bracket, and let $\bar{w} \in \widetilde{A}_{n-1} / A_{n-1}$ denote its corresponding dominant alcove. It is immediate then the fact that $w=\alpha^{B}\left(\bar{w}_{k}\right)$.

We are now in position to describe the bijection $\psi: \mathcal{R}^{m}\left(B_{n}\right) \rightarrow \mathcal{R}^{m}\left(A_{n-1}\right) \times\{1, \ldots, m n+1\}$. For a dominant alcove $R \in \mathcal{R}^{m}\left(B_{n}\right)$ we set $\psi(R)=(w, i)$, where $w$ and $i$ are obtained similarly to the type $C$ case, by applying Propositions 3.8 and 3.9 We leave it to the reader to check that $\phi$ defines a bijection between the sets $\mathcal{R}^{m}\left(B_{n}\right)$ and $\mathcal{R}^{m}\left(A_{n-1}\right) \times\{1, \ldots, m n+1\}$.

Corollary 3.10 The map $\bar{\psi}: \mathcal{R}^{m}\left(B_{n}\right) \rightarrow \mathcal{R}^{m}\left(A_{n-1}\right)$, defined by $\bar{\psi}(R)=\operatorname{pr}_{1}(\psi(R))$, is a surjection. 


\subsection{Examples}

Consider the dominant region $R$ of $\operatorname{Shi}^{3}\left(A_{3}\right)$ given in Example 2.4 Then $w=\left[4^{-3}, 1^{-1}, 2^{2}, 3^{2}\right] \in$ $\mathcal{A}\left(A_{n-1}\right)$ is its representative alcove. We will compute the type $C_{4}$ and $B_{4}$ Shi tableaux which correspond to the pairs $(R, 6)$ and $(w, 6)$. Proposition 3.5 implies that

$$
K_{3}(w)=\{-11,-10-7,-6,-3,-2,1,5,8,9,12,13,17\},
$$

thus $k_{6}=-2=2+4(-1)=2^{-1}$. Therefore $w_{-2}=\left(1^{2}, 2^{-3}, 3^{-2}, 4^{1}\right)$ and the type $C$ and $B$ expansion have vector of levels, respectively, $\vec{n}\left(\alpha^{C}\left(w_{-2}\right)\right)=(2,-3,-2,1,-1,2,3,-2)$ and $\vec{n}\left(\alpha^{B}\left(w_{-2}\right)\right)=$ $(2,-3,-2,1,0,-1,2,3,-2)$. Hence, $\alpha^{C}\left(w_{-2}\right)=\left[2^{-3}, 3^{-2}, 8^{-2}, 5^{-1}, 4^{1}, 1^{2}, 6^{2}, 7^{3}\right]$ and $\alpha^{B}\left(w_{-2}\right)=$ $\left[2^{-3}, 3^{-2}, 8^{-2}, 6^{-1}, 5^{0}, 4^{1}, 1^{2}, 7^{2}, 8^{3}\right]$. By applying Equation (2) we obtain respectively the tableau:

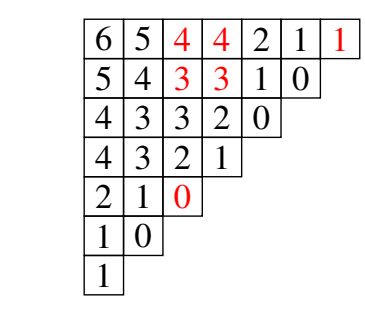

$$
\left[2^{-3}, 3^{-2}, 8^{-2}, 5^{-1}, 4^{1}, 1^{2}, 6^{2}, 7^{3}\right]
$$

and

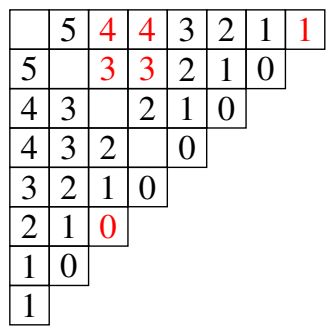

$$
\left[2^{-3}, 3^{-2}, 9^{-2}, 6^{-1}, 5^{0}, 4^{1}, 1^{2}, 7^{2}, 8^{3}\right]
$$

Now, by replacing with 3 all entries of the tableaux which are greater than 3 , we obtain the Shi tableaux of the corresponding dominant regions. Note that the red entries in the brackets and the tableaux generate respectively the bracket and tableau of $w$. We complete this section with an illustration of the map of Corollary 3.7 (Figure 3). Remark that $\operatorname{Shi}^{2}\left(A_{1}\right)$ has only three dominant regions, which have Shi tableau $[0],[1]$ and [2]. In each dominant region of $\operatorname{Shi}^{2}\left(C_{2}\right)$ it is written the dominant region of $\operatorname{Shi}^{2}\left(A_{1}\right)$ to which it is projected.

\section{Dominant regions and lattice paths}

In this section we prove Corollary 1.2 We recall that an $N-E$ lattice path is a path in the lattice grid $\mathbb{Z} \times \mathbb{Z}$ having north steps $(0,1)$ and east steps $(1,0)$. Given $n, m \geq 1$, we denote by $\mathcal{L}_{n}^{m}$ the set of $N-E$ lattice paths from $(0,0)$ to $(n, m n)$. An $m$-Dyck path of height $n$, is a path of $\mathcal{L}_{n}^{m}$ with the additional property that it does not go below the line $x=m y$. We denote by $\mathcal{D}_{n}^{m}$ the set of such paths, and remark that $\left|\mathcal{L}_{n}^{m}\right|=\left|\mathcal{D}_{n}^{m}\right|(m n+1)$. We will provide a natural bijection between the sets $\mathcal{L}_{n}^{m}$ and $\mathcal{D}_{n}^{m} \times\{1, \ldots, m n+1\}$. For this, we need to introduce some notation. Every $p \in \mathcal{L}_{n}^{m}$ can be uniquely determined by the step sequence $\left(s_{1}, \ldots, s_{n}\right)$, where each $s_{i}$ denotes the number of east steps occurring before the $i$-th north step. Thus we can write $p=\left(s_{1}, \ldots, s_{n}\right)$ as well. We note that $0 \leq s_{1} \leq \cdots \leq s_{n} \leq n$, and $p \in \mathcal{D}_{n}^{m}$, if and only if $s_{i} \leq m i$ for every $1 \leq i \leq n$. For example, for the path $p=E E N N E N E E E$ of $\mathcal{L}_{3}^{2}$ we can write $p=(2,2,3)$.

Definition 4.1 Let $p=\left(s_{1}, \ldots, s_{n}\right) \in \mathcal{L}_{n}^{m}$. For $0 \leq i \leq n-1$ we set $d_{i}(p)$ to be the path in $\mathcal{L}_{n}^{m}$ with step sequence $\left(0, s_{i+2}-s_{i+1}, \ldots, s_{n}-s_{i+1}, \bar{s}_{1}-s_{i+1}, \bar{s}_{2}-s_{i+1}, \ldots, \bar{s}_{i}-s_{i+1}\right\}$, where $\bar{s}_{j}=s_{j}+m n+1$. 


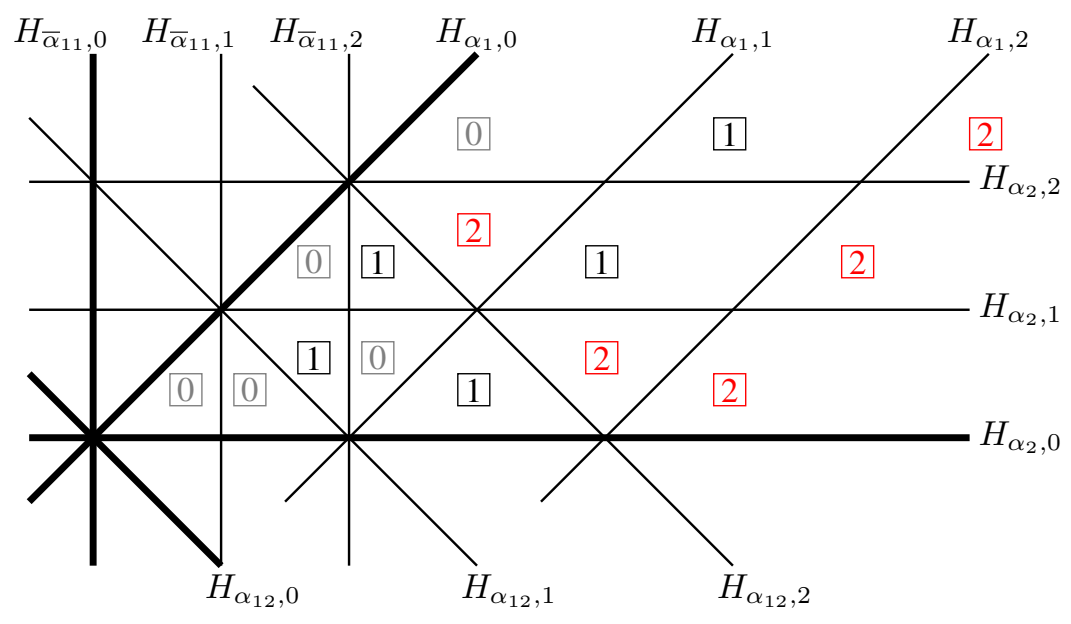

Fig. 3: The projection of the dominant regions of the arrangement $\operatorname{Shi}^{2}\left(C_{2}\right)$ to those of $\operatorname{Shi}^{2}\left(A_{1}\right)$.

The proof of the following proposition is omitted.

Proposition 4.2 Let $p \in \mathcal{L}_{n}^{m}$. Then there exists a unique index $0 \leq i_{*} \leq n-1$, such that $d_{i_{*}}(p) \in \mathcal{D}_{n}^{m}$.

Proof of Corollary 1.2 (sketch): In view of Theorem 1.1, and [7, Theorem 3.1], it is enough to construct a bijection between the sets $\mathcal{L}_{n}^{m}$ and $\mathcal{D}_{n}^{m} \times\{1, \ldots, m n+1\}$. For that we will use an idea from [10, Chapter 1.4]. Let $p=\left(s_{1}, \ldots, s_{n}\right) \in \mathcal{L}_{n}^{m}$, and $0 \leq i_{*} \leq n-1$ as in Proposition 4.2 We define $h: \mathcal{L}_{n}^{m} \rightarrow \mathcal{D}_{n}^{m} \times\{1, \ldots, m n+1\}$ by letting $h(p)=\left(d_{i_{*}}(p), s_{i_{*}}+1\right)$. Note the fact that the map $h$ is well-defined is a direct consequence of Proposition 4.2. We leave it to the reader to check that $h$ is a bijection.

Example 4.3 Let $p=(5,5,9,12) \in \mathcal{L}_{4}^{3}$. Then $d_{0}(p)=(0,0,4,7), d_{1}(p)=(0,4,7,13), d_{2}(p)=$ $(0,3,9,9)$, and $d_{3}(p)=(0,6,6,10)$. Moreover, we have $i_{*}=0$, and $s_{i_{*}}=5$, thus $h(p)=((0,0,4,7), 6)$. Figure 4 illustrates the lattice path $P R$ constructed in the proof of Proposition 4.2. The dashed squares represent path $d_{i_{*}}(p)$. In view of the bijection given in [7, Theorem 3.1], the Dyck path $(0,0,4,7)$ corresponds to the dominant region whose Shi tabeau is given in Example 2.4. Moreover, the path $p$ corresponds to the region that has representative alcove the 3-minimal alcove $\alpha^{C}\left(w_{-2}\right)$ of Section 3.2

Acknowledgments. The authors are grateful to Eli Bagno for helpful discussions and to Philippe Nadeau for bringing [10] to their attention.

\section{References}

[1] Armstrong, D., Hanusa, H., \& Jones, B. 2014. Results and Conjectures on simulateneous core partitions. European J. Combin, 41, 205-220.

[2] Athanasiadis, C.A. 2005. On a refinement of the generalized Catalan numbers for Weyl groups. Trans. Amer. Math. Soc., 357, 179-196. 


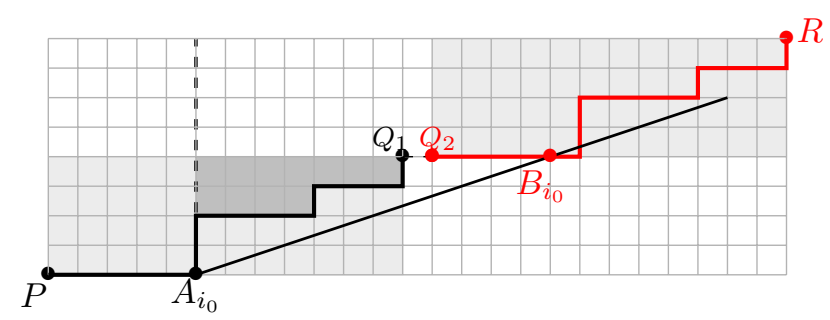

Fig. 4: The construction of Proposition 4.2 for the path $p=(5,5,9,12) \in \mathcal{L}_{4}^{3}$.

[3] Berg, C., Jones, B., \& Vazirani, M. 2009. A bijection on core partitions and a parabolic quotient of the affine symmetric group. J. Combin. Theory Ser. A, 116(8), 1344-1360.

[4] Björner, A., \& Brenti, F. 2005. Combinatorics of Coxeter Groups. Graduate Texts in Mathematics, vol. 231. Springer, New York.

[5] Fishel, S., \& Vazirani, M. 2010. A bijection between dominant Shi regions and core partitions. European J. Combin, 2087-2101.

[6] Fishel, S., Tzanaki, E., \& Vazirani, M. 2013a. Counting Shi regions with a fixed separating wall. Annals of Comb., 17(4), 671-693.

[7] Fishel, S., Kallipoliti, M., \& Tzanaki, E. 2013b. Facets of the generalized Cluster complex and regions in the extended Catalan arrangement of type A. Electron. J. Combin., 20(4).

[8] Hanusa, C.R.H., \& Jones, B.C. 2012. Abacus models for parabolic quotients of affine Weyl groups. J. Algebra, 361, 134-162.

[9] Kallipoliti, M., \& Tzanaki, E. 2014. Bijections of dominant regions in the $m$-Shi arrangements of type $A, B$ and $C$. In preparation.

[10] Mohanty, S. G. 1979. Lattice path counting and applications. New York: Academic Press [Harcourt Brace Jovanovich Publishers]. Probability and Mathematical Statistics.

[11] Shi, J-Y. 1987. Alcoves corresponding to an affive Weyl group. J. London Math. Soc., 35, 42-55.

[12] Shi, J-Y. 1997. The number of $\oplus$-sign types. Quart. J. Math. Oxford, 48, 93-105.

[13] Shi, J-Y. 1999. On two presentations of the affine Weil groups of classical types. J. Algebra, 221, 360-383.

[14] Shi, Jian Yi. 1986. The Kazhdan-Lusztig cells in certain affine Weyl groups. Lecture Notes in Mathematics, vol. 1179. Berlin: Springer-Verlag.

[15] Stanley, Richard P. 1998. Hyperplane arrangements, parking functions and tree inversions. Pages 359-375 of: Mathematical essays in honor of Gian-Carlo Rota (Cambridge, MA, 1996). Progr. Math., vol. 161. Boston, MA: Birkhäuser Boston. 